U. S. Geological Survoy

Tater Resources Division

P. 0. Box 138

Rolla, Missouri

Spring Rivor Basin

Low-ilater Study

Jct. 6, 7, 1953

By liarry C. Bolon

$$
54.24
$$

Open-file report. Not reviewed for conform co with the editorial standaras of the Geological Survey. 
U. S. Goological Survey

Water Resources Division

P. 0. Box 138

Rolla, Missour1

Spring River Besin

Low-Water Study

Oct. 6, 7, 1953

Because of the severe drought conditions and the turmoil brought about by it, observations of discharge were made at 46 points within that part of the Spring River basin that lies within the State of Missouri. Three of these measurements were made at regular gaging stations.

Two partios were formed and did all the measuring during the two-day periad 0ot. 6 and 7. The study was affected somewhat by rain that fell rather soattered over the basin 0ct. 3 and 4. This break in the drought caused the plowing streams to increase some in flow but did not cause many dry streams to flow. In spite of this precipitation it was decided to go ahead with the study as it would serve well to determine the general source of the flow in the besin, would determine which streams are intermittent, and would go a long way in evaluating the prime low-water resources within the area.

The Spring River basin, particularly around the Carthage-Joplin area is faced with supply and health problems probably more than any other area within the State, as Spring River, Center Croek, and Shoal Creek are all used heavily for various purposes.

Only three regular gaging stations are maintained in the Missouri portion of the Spring River besin, and the only non-regulated ore that existed during the drought period of 1934-36 is the station on Spring River near Waco, Mo. This station was established in April 1924 and the minimun flow on record was $11 \mathrm{cfs}$ July 24, 1934. The low on Oct. 7, 1953, was measured as 26.6 cfs. Before the rain on Oct. 3 the flow was as low as about 13 ofs at Haco, which indicates that in the Spring River bosin the 1953 drought was nearly as severe as the droughts of 1934 and 1936 although in all instances regulation affects the low-water flow and therefore makes such a comparison rather questionable. Mills, pumpage, and other slight regulations make it rather difficult to compare droughts by stream flov in this area.

The flow at various points along the main stems of Spring River, Center Creek, and Shoal reck are as follows:

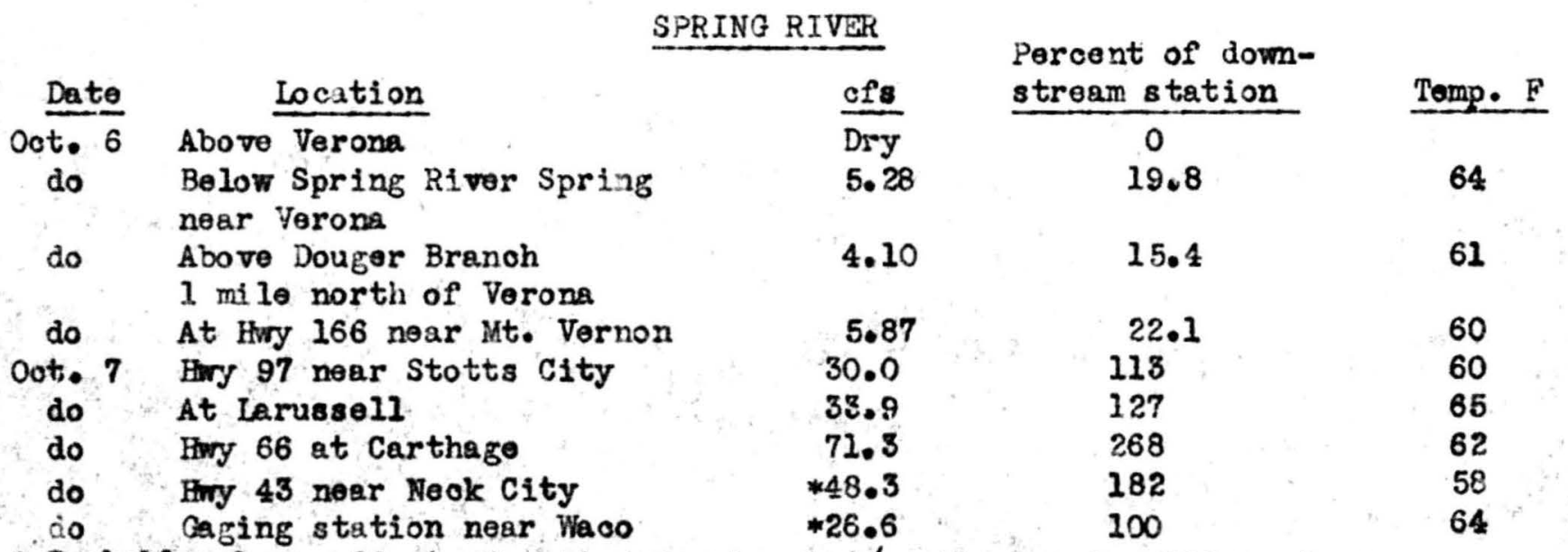

* Probably shows affect of upstream rains and/or storage in mill ponds. 


\section{CENTER CREER}

\begin{tabular}{|c|c|c|c|c|}
\hline Date & Location & cfs & stream station & Temp. F \\
\hline Oct. & Hory 97 near Stotts City & Dry & 0 & \\
\hline do & Above Clarkston Spring & Dry & 0 & \\
\hline do & $\frac{1}{2}$ mile above Dry Velley & 6.95 & 31.4 & 56 \\
\hline do & Howy 166 at Sarcoxio & 10.1 & 45.7 & 56 \\
\hline do & Hwry 37 at Reeds, Mo. & 9.76 & 44.1 & 58 \\
\hline do & $\begin{array}{l}\text { Hwy } 71 \text { about } \frac{1}{4} \text { mile below } \\
\text { Jones Creak and near Carthage }\end{array}$ & 14.3 & 64.7 & 57 \\
\hline do & Hwy 57 at Carl Junction & 22.1 & 100 & 60 \\
\hline
\end{tabular}

SHOAL CREEK

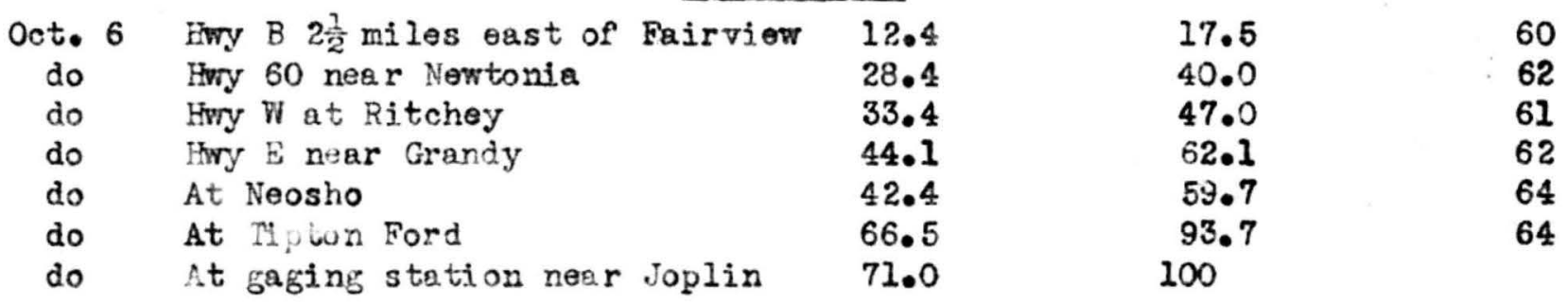

Below is a list of discharge measurements and observations of no flow mace within the basin $0^{\prime}$ Spring River, Center Creek, and Shoal Creok Oct. 6 and 7, 1953, listed in downstream order.

Harry C. Bolon

District Engineer

No vember 12,1953 


\section{Spring River Basin}

Dete

1953

ct. 6 Spring River

6 Spring River Spring

6 Spring River

6

do

6

Douger Branch Spring River

6 Spring River

6 Honey Creek

Spring Fiver

Honey Craek

Spring River

Neosho Piver

Spring River

Neosho River

Neosho River

6 Honey Creek

\section{Lesetion}

Stege

Flow cf8

On east line of $\mathrm{NE} \frac{3}{4} \mathrm{NW} \frac{3}{4} \mathrm{sec} .28, \mathrm{~T}_{0}$ $26 \mathrm{~N} \bullet, R_{\bullet} 26 \mathrm{~W}$, , at ford I mile Dry above Browning Hollow and 2 miles southeast of Verona, Mo.

$N W \frac{1}{4} N W_{\frac{1}{4}}^{2}$ sec. 28, T. $26 \mathrm{~N}_{\bullet}, \mathrm{R}_{0} 26 \mathrm{~W}_{\bullet}, \quad-$

$500 \mathrm{ft}$ from Spring River and $1-3 / 4$

miles southeast of Verona, No.

On west line SW $\frac{1}{4} S W \frac{1}{4}$ sec. 2l, T. $26 * 10.60$

N., R. 26 W., at county road $\frac{1}{4}$ mile above Hory $60, \frac{1}{2} \mathrm{mile}$ above Browning Hollow, and $1 \frac{1}{2}$ miles southeast of Verona, Mo.

$\mathrm{SE}_{\frac{1}{4}}^{\frac{1}{4}}$ sec. 8, T. $26 \mathrm{~N}, \mathrm{R}, 26 \mathrm{~W}$, at county road $500 \mathrm{ft}$ above Douger Branch, and 1 mile north of Verona, Mo.

$\mathrm{SE}_{4}^{\frac{1}{4}} \mathrm{sec} \cdot 8, \mathrm{~T}_{0} 26 \mathrm{~N}, \mathrm{R}_{\bullet} 26 \mathrm{~W}$, , at - Dry Hwy $P, \frac{1}{4}$ mile above mouth and 1 mile north of Verone, Mo.

SW $\frac{2}{2}$ sec. 2, T. 27 N., R. 27 W., at $* 19.07$ 5.87 Hwy 166, about $\frac{1}{2}$ mile above Honey Creek and 3 miles southwest of Mount Vernon, Mo.

On east line of NE $\frac{1}{4} N W \frac{1}{4}$ sec. 35, $\cdot 38$

T. $27 \mathrm{H}, \mathrm{R}_{0} 25 \mathrm{~W}$, , at county highway bridge at north edge of Marionville, Mo.

$\mathrm{NE} \frac{-1}{4} W_{4}$ sec. 22, T. 27 N., R. 25 1.87 W., 500 ft from Honey Creek and $2 \frac{2}{2}$ miles northwest of Marionville, Mo. 
Oct. 6 Honey Creek Spring River

6 Truitt Creek Spring River

6 Will lams Creek Truitt Creek

7 Stahl Creek Spring River

7 Spring River Neosho River

7

do

do

7 White Oak Creek Spring River

7 Spring River Neosho River

7

do

do

7 North Fork Spring River

Spring River

7. Coon Creek

North Fork Soring River

7 Dry Creek
SW $\frac{1}{6}$ sec. 2, T. 27 N., R. 27 W., - Dry at Highway 166 , about $\frac{1}{3}$ mile above mouth and 3 miles southwest of Mount Vernon, Mo.

NW $\frac{1}{6}$ SN sec. 23, T. 28 N., R. 27 W., at county road $1 \frac{1}{2}$ miles above Williams Creek and $2 \frac{1}{2}$ miles northwest of Mt. Vernon. Mo.

NWt sw sec. 30, T. 28 N., R. 26 W., at bridge by State Sanitarium, $\frac{1}{4}$ mile above Frisco R.R., at north edge of Mt. Vernon, Mo.

At USGS gaging station near Miller, Mo.

SW sec. 13, T. $28 \mathrm{~N} ., R_{0} 28 \mathrm{~W} ., \quad * 21.5430 .0$ at State Highway 97, about 2 mil es north of Stotts City. Mo.

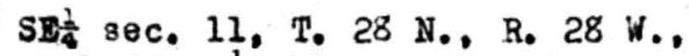
at bridge $\frac{1}{2}$ mile nor theast of Larussell, Mo.

SW corner sec. 6, T. 28 N., R. 29 $W_{1}$, at State Highway 37 about $1 \frac{1}{8}$ miles above mouth and $2 \frac{1}{3}$ miles southwest of Avilla, Mo.

SE- sec. 33, T. $29 \mathrm{~N} .$, R. 31 W., at U.S. Highway 71 at north edge of Carthage, Mo.

On line between sec. 1 and 2 , $.25 .42 \quad 48.3$ T. 29 N., Ro 33 W., at Highway 43 , about 3 miles abov North Fork and 3 miles nor thwest of Neck City, Mo.

SW corner sec. 13, T. $31 \mathrm{~N}$. , $R 31$ W.. at U. S. Highway 71 about $4 \frac{1}{6}$ miles south of Lamar, Mo.

NWt at U.S. Highway 71 about 1 mile north of Jasper, Mo.

On line between sec. 3 and 4, T. $29 \mathrm{~N}_{0}, \mathrm{~K}_{0} 31 \mathrm{~W}$. , at U.S. Hi ghway il about $\frac{1}{3}$ mile north of Cary town, Mo. 
7 North Fork Spring River

7 Spring River
Spring River

Neosho River
On line between sec. $I$ and 2 , T. 29 N., R. 33 W., at State Highway 43 about $\frac{1}{4}$ mile below Little Spring River and 3 miles northwest of Neck City. Mo.

At regular gaging station near Waco, Mo.

\section{Center Creek Basin}

7 Center Creek

Spring River

तอ

7. do io
SW corner sec. 22, T. 27 N., R. $28 \mathrm{~W}$., at State Highway 97 about 5 miles southwest of Stotts City, Mo.

7. Clarkson Spring venter Crsek

7 Center Creek Spring River

7 EIi Iynn Spring Center Creek

7 Dry Valley

Center Creek

7 Center Creek

Spring River

7

do

7. Jones Creek west of Pierce City. Mo.

Ste sec. 17, T. 27 N., R. 28 W., 7 miles northwest of Pierce city, Mo.

On west line of $\mathrm{Nat}$ SWt sec. 23 , T. 27 N., R. 29 W., at old ford $\frac{1}{2}$ mile above Dry Valley Branch 600 feet from Center Creek and at mouth and 3 miles north of ientworth, Mo. at U.S. Highway 166 at east edge of Sarcoxie, Mo.

On west line of $\mathrm{SW}_{\frac{1}{4}} \mathrm{SW} \frac{1}{4}$ sec. 1 , T. 27 N., R. 30 W. , on State Highway 37 , about $2 \frac{1}{2}$ miles south of Reeds, Mo.

Center Creek

On south line of Sistit sec. 35 ,
SEs $\frac{1}{4}$ Set sec. 17, T. 27 N., R. 28 W. Just upstream from mouth of Clarkson Spring Branch and 7 miles northand 3 miles north of Wentworth, Mo.

SEd SW $\frac{1}{4}$ sec. 23, T. 27 N., R. 29 W., 2 3/4 miles north of Wentworth. Mo.

Dry

Dry

SESEE⿱

SW $W$ W sec. 9. T. 27 N., R. 29 W., T. 28 N.. R. 31 W., at county road $\frac{1}{a}$ mile below Jenking Creek and 3 iniles northwest of Parshley, Mo. 
7 Center Creek

7 Grove Creek

7 Center Creek
Spring River

Center Creek

Spring River
On west line of NWt sec. 34 ,

T. 28 N., R. 31 W., at U.S. Highway

71 about $\frac{1}{4}$ mile below Jones Creek and 5 miles south of Carthage. Mo.

In extreme SW corner of $S E \frac{2}{4}$ sec. 25 ,

T. 28 N., R. 32 W., at county road

$3 / 4$ mile upstream from mouth and

2 miles north of Scotland, Mo.

Nes sec. 9, T. 28 N., R. 33 W., at 17.75

State Highway 57, at Carl Junction, Mo.

Shoal Creak Basin

6 Shoal Creek

Spring River

On north line NWt sec. 10, T. 24 N.\$19.38 12.4

R. 29 W., on County Highway $B$ about

1 mile south of Pioneer and $2 \frac{1}{2}$

miles east of Fairview, No.

6 do

do

$N W_{4}^{-1} N W_{4}^{\frac{1}{4}}$ sec. 5, T. $25 \mathrm{H}, \mathrm{R}_{0} 29 W_{0}, 17.1428 .4$ at U.S. Highway 60 about $1 \frac{1}{7}$ miles

above Clear Creek and $3 \frac{1}{2}$ miles

northeast of Newtonia, Mo.

6 Kelly Creek

Clear Creek

SW- SBt sec. 32, T. 26 N., R. 27 W..

at mouth just above old highway

Dry

bridge 500 feet above U.S. Highway

60 at southwest edge of Monett. Mo.

6 Clear Creek

Shoal Creek

Setsits sec. 31, T. 26 N., R. 27 W., at U.S. Highway 60 , about 500 feet below Kelly Creek at southwest edge of Monett. Mo.

do

NW $\frac{1}{4}$ sec. $28 .$, T. 26 N., R. 28 W.. 13.39

at new highway bridge at south edge

of Pierce City, Mo.

6 do

do

S3: 5 Sw sec. 30, T. 26 N., R. 29 W. about 300 feet above mouth and $2 \frac{1}{2}$ miles east of Ritchey, Mo.

Dry

Swts sec. 26, T. 26 s., R. 30 W., $* 23.60: 33.4$ at now bridge on County Highway W.. 500 feet below old steel bridge, 1,000 feet below mill and dam, and tile southwest of Ritchey. Mo. 

at County Highway $\mathrm{E}, 3 / 4 \mathrm{mile}$ above Frisco R.R., and 2 miles north of Granby, Mo.

6

do

do

do

6

do

do

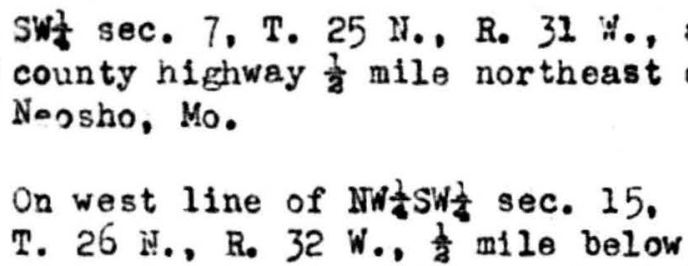

Cedar Creek and at Tipton Ford, Mo.
At USGS gaging station above Joplin, Mo.
66.5

3.58

71.0

* Distance down to water surface from reference point.

e - Estinated.

a - Probably slightly affected by water belng stored in mill pond during measurement.

0 - No flow with pools of water in channel.

Dry - Dry channel. No water in sid. 


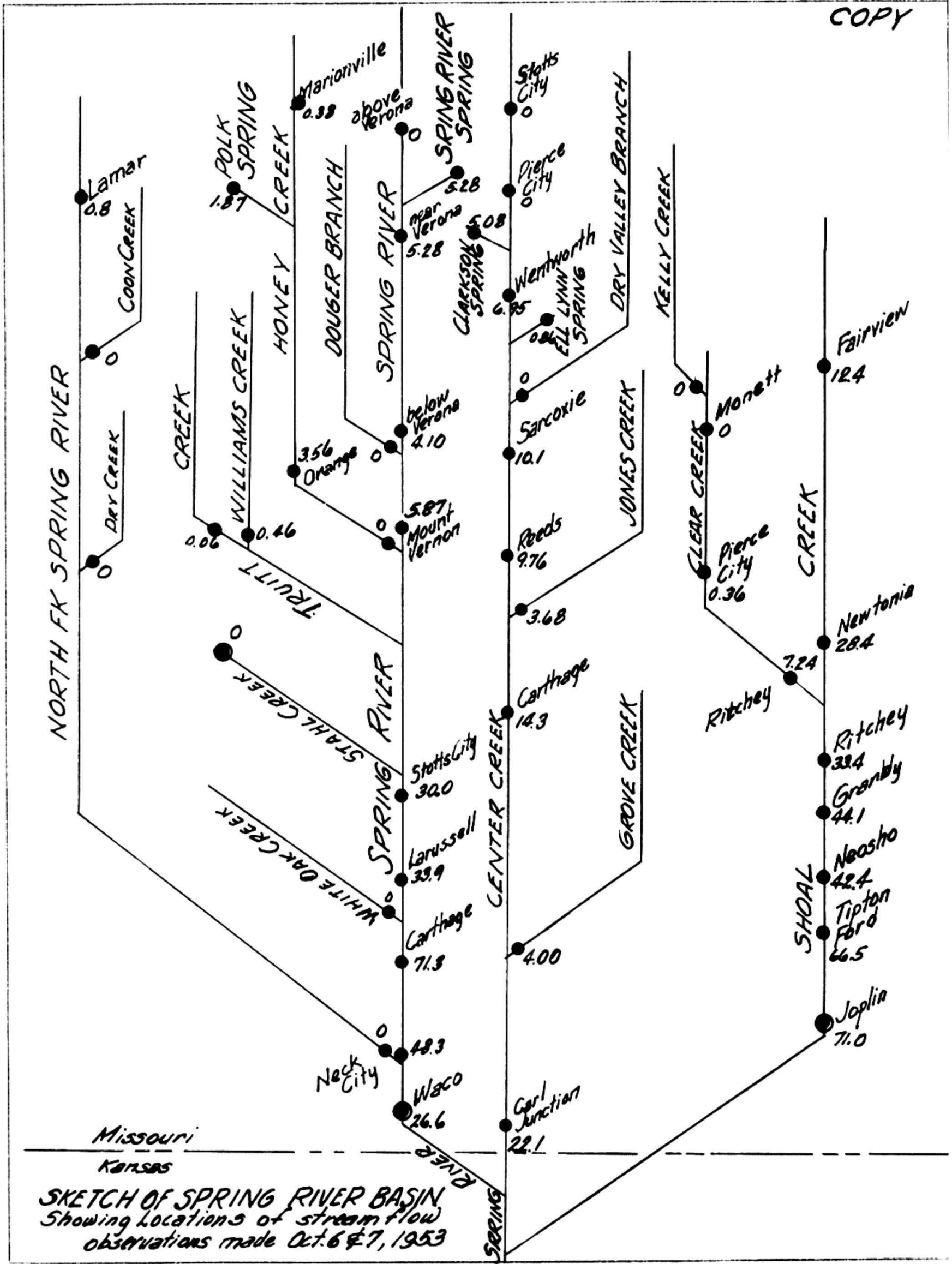

